\title{
Research Paper \\ Chemical, Sensory and Survival Properties of Lactobacillus Plantarum in Peach Juice
}

\section{Anahita Hosseinpour ${ }^{1}\left[\right.$ (), Saeed Shahsavari ${ }^{2,3}{ }^{(0)},{ }^{*}$ Razzagh Mahmoudi ${ }^{4}$ (1)}

1. Student Research Committee, School of Health, Qazvin University of Medical Sciences, Qazvin, Iran.

2. Health Products Safety Research Center, Qazvin University of Medical Sciences, Qazvin, Iran.

3. Department of Epidemiology and Biostatistics, School of Public Health, Tehran University of Medical Sciences, Tehran, Iran.

4. Medical Microbiology Research Center, Qazvin University of Medical Sciences, Qazvin, Iran.

\begin{tabular}{|l|l|l|l|l}
\hline $\begin{array}{l}\text { Use your device to scan } \\
\text { and read the articl online }\end{array}$ & Citation Hosseinpour A, Shahsavari S, Mahmoudi R. Chemical, Sensory and Survival Properties of Lactobacillus Plantarum in Peach \\
Juice. The Journal of Qazvin University of Medical Sciences. 2019; 23(4):342-351. https://doi.org/10.32598/JQUMS.23.4.342
\end{tabular}

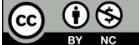

Received: 30 Dec 2018 Accepted: 15 Jun 2019 Available Online: 01 Oct 2019

Keywords:

Lactobacillus Plantarum, Probiotic, Peach

\section{ABSTRACT}

Background Nowadays, the consumption of probiotic food products has become common. Since the consumption of some dairy products is not allowed for some people, there is a need for some alternative foods. Fruits are suitable for the production of probiotic drinks due to the lack of any adverse effects. Objective This study was aimed to investigate the chemical and sensory properties and the survival of Lactobacillus plantarum (L. plantarum) bacteria in peach juice.

Methods This experimental study was conducted in Food Safety Laboratory of Qazvin University of Medical Sciences in autumn 2018. The peach juice samples were prepared from San Inch Company in Iran and kept in refrigerator to be used for the inoculation of $L$. plantarum. On days 1, 3, 5, 7 and 10, the survival of this bacterium, and changes in its $\mathrm{pH}$ and acidity were measured in two test and control groups. At the $10_{\text {th }}$ day, the samples underwent evaluation. Data were analyzed in SPSS V. 23 software using independent $t$-test and ANOVA at the significant level of $P<0.05$.

Findings After 10 days, the number of L. plantarum was reduced from 8.7 to 8.4 log cycles. With the increase of storage time, $\mathrm{pH}$ value significantly decreased and acidity increased $(\mathrm{P}<0.05)$. The sensory properties of the probiotic product were not significantly different from that of non-probiotic product and were acceptable for the consumer $(P>0.05)$.

Conclusion L. plantarum has a good shelf life in peach juice. Therefore, it can be concluded that peach juice is a suitable product for producing probiotic juice by adding L. plantarum.

\section{Extended Abstract}

\section{Introduction}

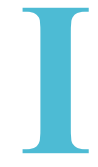

nadequate nutrition can cause various diseases. One way to prevent these diseases is to eat probiotic foods [1]. Probiotics are microorganisms that deploy to different parts of the body (especially to the gut, as natural flora) to maintain and im- prove balance in intestinal microflora (between beneficial and harmful microorganisms) and create health-promoting properties for the host [2]. Currently, most probiotic products are dairy products, but the demand for non-dairy probiotic products has increased in recent years [4]. People with lactose intolerance and vegetarians have reduced the consumption of dairy products. If no suitable alternative to dairy probiotic products is found for vegetarians, probiotics with very high medicinal properties will gradually lose

* Corresponding Author:

Razzagh Mahmoudi

Address: Medical Microbiology Research Center, Qazvin University of Medical Sciences, Qazvin, Iran.

Tel: +98 (912) 7868571

E-Mail: r.mahmodi@yahoo.com 
their place among a wide range of people [2]. Fruits and vegetables are rich in antioxidants, vitamins, and minerals. Besides, fruits and vegetables do not have the disadvantages reported for dairy products that prevent them from being consumed by a specific group of people. Therefore, they are useful alternatives for the production of probiotic products [4]. Peach juice has the highest market share in terms of juice production and has been highly considered for its nutritional value due to its high vitamin $\mathrm{A}$, vitamin $\mathrm{C}$, and potassium contents [7]. This study aimed to investigate the survival of Lactobacillus plantarum (L. plantarum) bacteria in peach juice and its effect on the chemical and sensory properties of the final product and, eventually, the production of probiotic peach juice.

\section{Materials and Methods}

The peach juice was prepared from Sunich Company in Iran and stored at $4^{\circ} \mathrm{C}$ until use. In the next step, $100 \mathrm{~mL}$ of the juice was transferred to each sterility test container. Two samples of juice were prepared each day; one sample was the control, and the other was inoculated with L. plantarum bacteria [10]. The samples were stored in refrigerator temperature, and then their living bacteria and $\mathrm{pH}$ was measured on days $1,3,5,7$, and 10 . After storage in the refrigerator for the mentioned days, cell viability was counted by standard plate count method with surface cultivation on MRS agar medium for $48 \mathrm{~h}$ at $37^{\circ} \mathrm{C}$ [16]. A pH meter was used to measure the $\mathrm{pH}$. To measure the acidity, $10 \mathrm{~mL}$ of the sample was poured into an Erlenmeyer flask and titrated with $0.1 \mathrm{~N} \mathrm{NaOH}$ in the vicinity of the phenolphthalein indicator to give a stable pink color. The acidity was calculated as:

$$
\text { Acidity }=(V \times N \times 0.064 \times 100) /(\text { Sample size })
$$

Treatment and control samples were evaluated on day 10 , with the presence of seven sensory examiners in terms of color, taste, and odor [9]. All tests were performed three times, and data were analyzed in SPSS V. 23 using independent $t$ test and ANOVA at a significant level of $\mathrm{P}<0.05$.

\section{Results}

According to the results, the initial $\mathrm{pH}$ of the treated sample and the control sample were 3.65, and 3.7, respectively, which changed to 3.1 and 3.3 at the end of the bacterial survival in the juice, respectively. The acidity of the treated sample and the control sample on the first day were 0.51 $\mathrm{g} / \mathrm{L}$ and $0.48 \mathrm{~g} / \mathrm{L}$, respectively. At the end of bacterial survival in peach juice, the acidity in the treated and control samples reached 0.7 and 0.76 , respectively. The number of bacteria in peach juice decreased from $8.7 \mathrm{log}$ cycles to
$8.4 \log$ cycles within 10 days after inoculation; i.e., it could maintain its viability. On day 10 , no significant differences were observed between the control and test samples in terms of color, taste, and odor $(\mathrm{P}>0.05)$. Thus the addition of L. plantarum to peach juice had no adverse effect on its sensory properties and was acceptable to the consumer.

\section{Conclusion}

The results of this study showed that the $\mathrm{pH}$ of the treated sample significantly decreased, but its acidity increased considerably. The number of probiotic bacteria decreased during storage but showed a good shelf life in the juice. The sensory properties of probiotic peach juice were not significantly different from the non-probiotic product. Khezri et al. [16] used fig juice as a base for inoculating Lactobacillus delbrueckii with inulin. The results showed that fig juice was a suitable environment for the survival of Lactobacillus delbrueckii, which is in agreement with the results of the present study.

Results of Totonchi et al. [4] showed that Lactobacillus acidophilus increased acidity during refrigerated storage, which is consistent with our results. Ghorbani et al. [11] reported the possibility of using Lactobacillus plantarum and Lactobacillus kunkeei isolated from honey in the preparation of probiotic pomegranate juice where the $\mathrm{pH}$ decreased significantly with the increase in storage time and probiotic population. This result is also consistent with our findings. According to the results of our study, peach juice with its nutrients can be a good environment for probiotic bacterial growth. L. plantarum had desirable probiotic properties according to standards. It also has good growth and viability and can be used to enhance the nutritional properties of peach juice.

\section{Ethical Considerations}

\section{Compliance with ethical guidelines}

The present study was approved by the Research Ethics Committee of Qazvin University of Medical Sciences (Code: IR.QUMS.REC.1397.160).

\section{Funding}

The present study received financial support from the Deputy for Research of Qazvin University of Medical Sciences.

\section{Authors' contributions}

All authors had equally contributed in preparing this paper. 
Conflicts of interest

The authors declared no conflict of interest.

Acknowledgements

The present article was extracted from a research proposal approved by Qazvin University of Medical Sciences. 


\title{
ارزيابى خصوصيات شيميايى، حسى و ماندكارى لاكتوباسيلوس بِلاتتاروم در آبميوه هلو
}

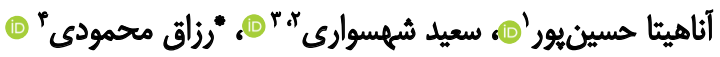

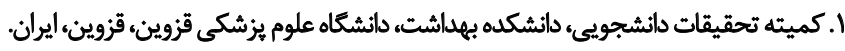

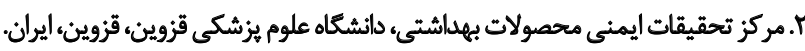

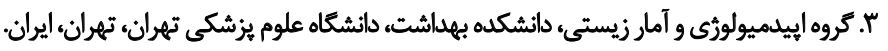 \\ F. مركز تحقيقات ميكروبشناسى يزشكى، دانشكاه علوم يزشكى قزوين، قزوين، ايران.
}

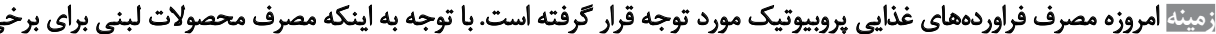

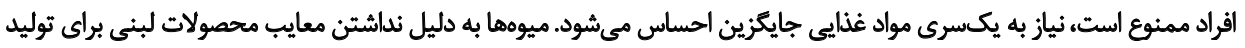
نوشيدنى هاي يروبيوتيك مناسب هستيند.

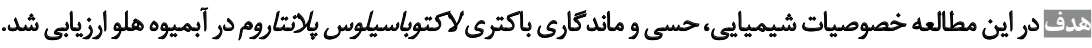

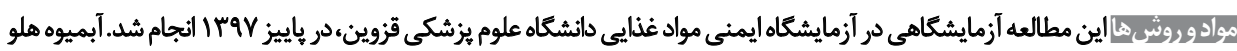

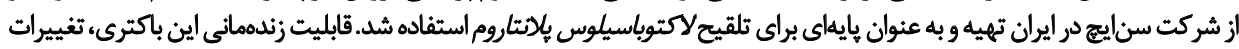

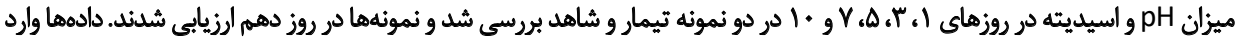

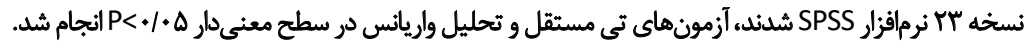

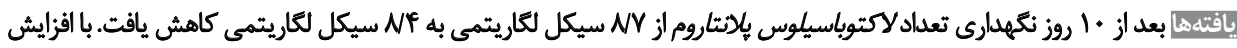

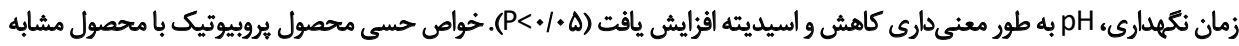

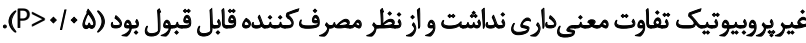

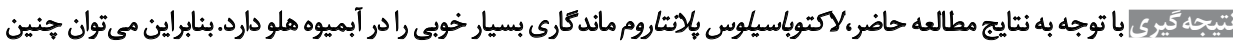

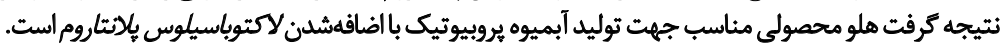

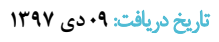

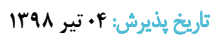

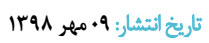

مىشوند. مهمترين ثروبيوتيكها به جنس هاى لاكتوباسيلوس'

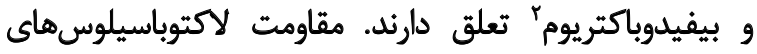

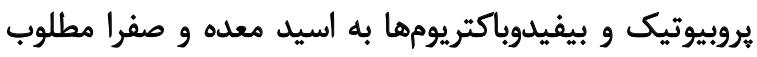

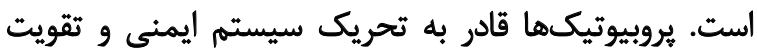

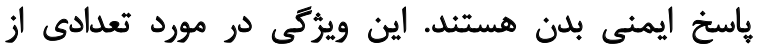

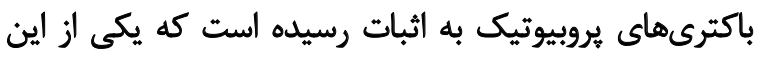

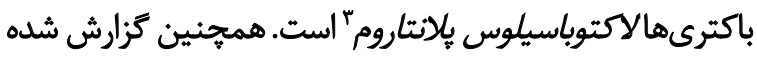

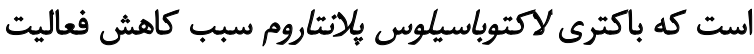

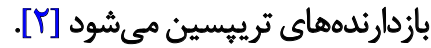
غذاى باكترىهاى يروبيوتيك، برىبيوتيك ناميده

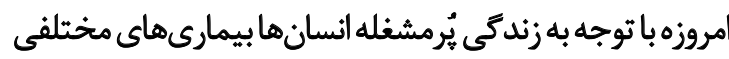

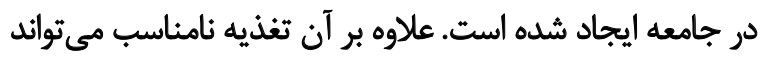

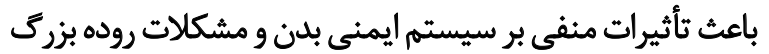

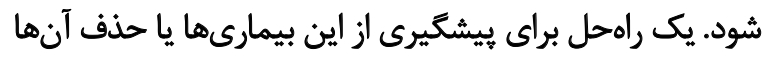

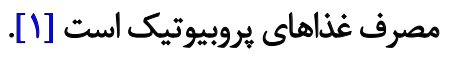

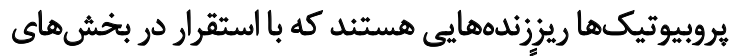

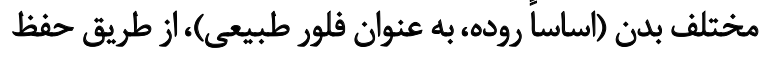

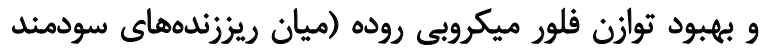
و زيانبخش)، سبب ايجاد خواص سلامتبخش برون مراى ميزبان 
يإنتاروم و لاكتوباسيلوس كانكئى" جداشده از عسل در تهيه

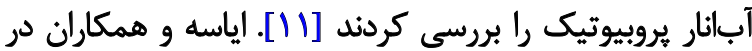

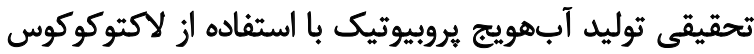

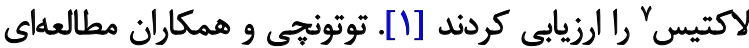

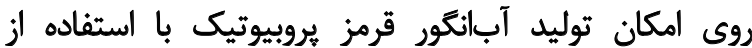

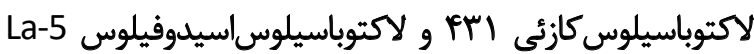

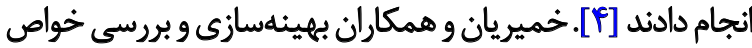

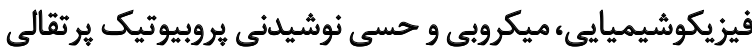

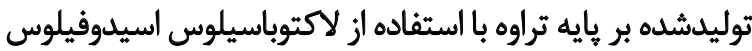

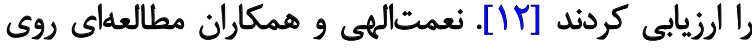

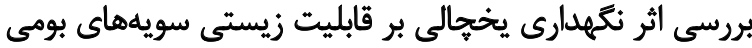

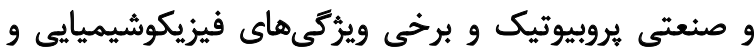

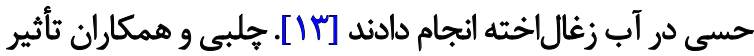

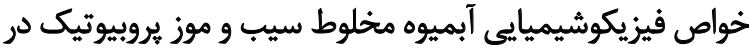

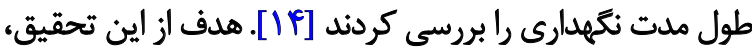

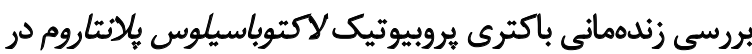

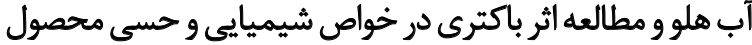
نهايى و درنهايت توليد آب هلو بروبيوتيك بودئي

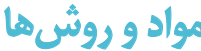

اين مطالعه آزمايشكاهى در آزمايشكاه ايمنى مواد غذايى

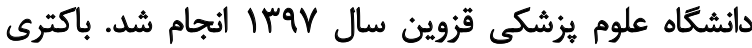

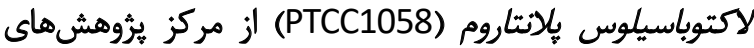

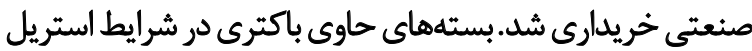

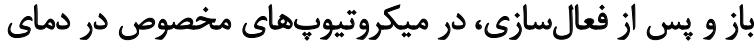

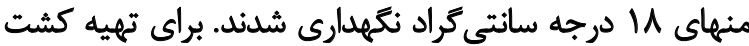

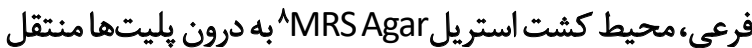

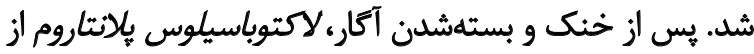

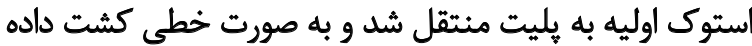

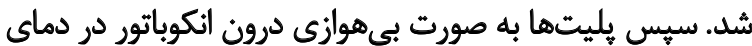

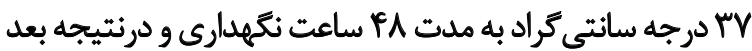

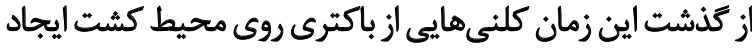

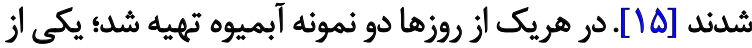

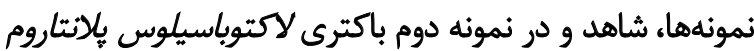

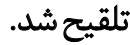

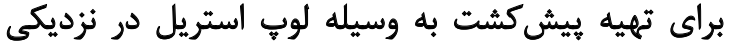

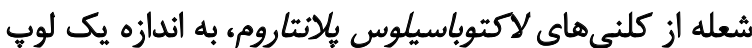

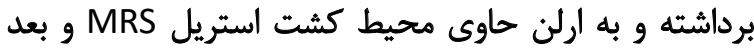

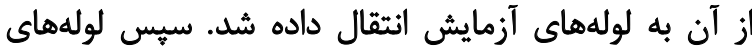

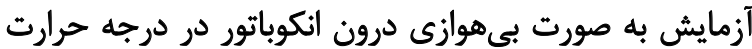

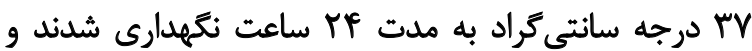

6. Lactobacillus caneci
مىشود. يرىبيوتيكها به رشد باكترىهاى مفيد از قبيل

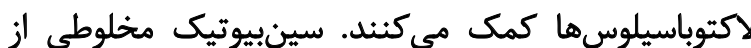

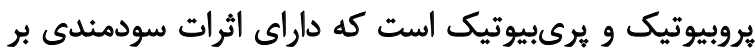

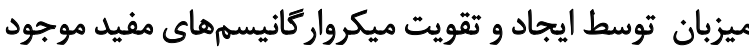

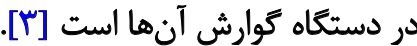

غذاهايى كه سبب بهبود سلامتى شده و مواد مغذى ضرورى فئمي

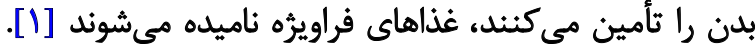

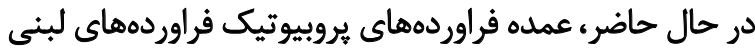

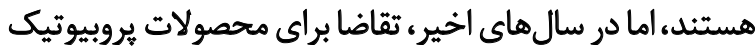

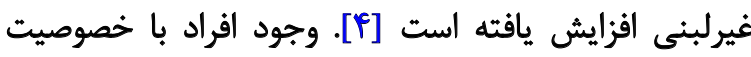

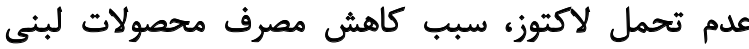

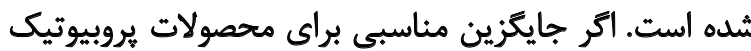

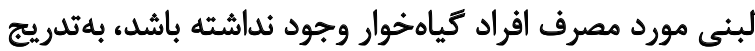

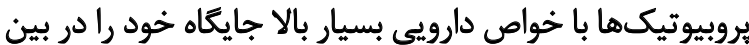

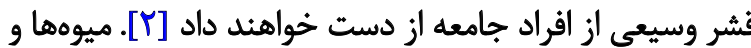

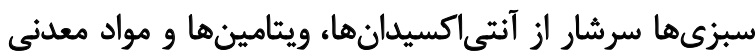

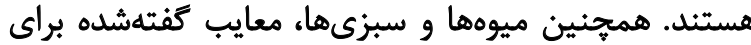

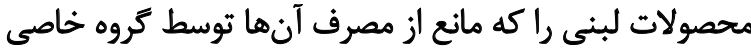

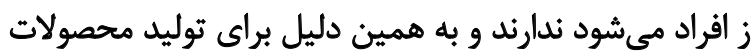

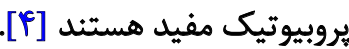

در سال هاى اخير تحقيقات متعددى در راستاى توليد آبميوه

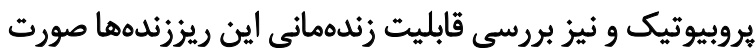

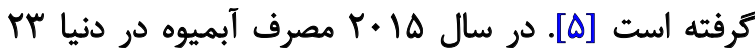

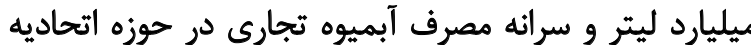

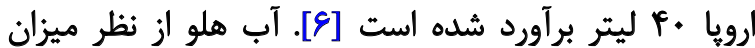

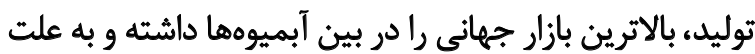

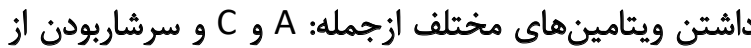

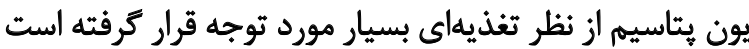

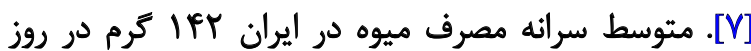

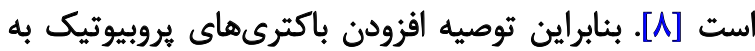

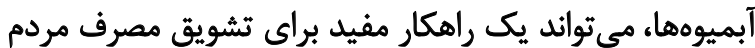
به مواد غذايى فراسودمند باشد.

تاكنون تحقيقات زيادى در ارتباط با فرايند توليد و خواص

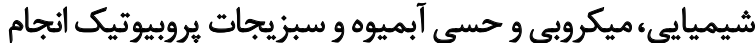

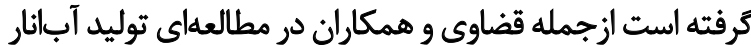

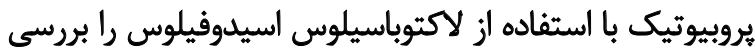

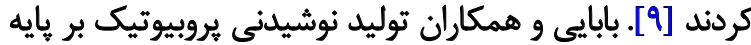

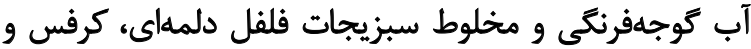

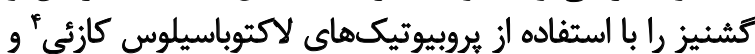

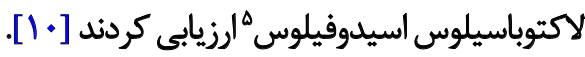
قربانى و همكاران در يرؤهى استفاده از لاكتوباسيلوس 
كه مىتواند به علت مرك جمعيتى از باكترىها و توليد اسيد

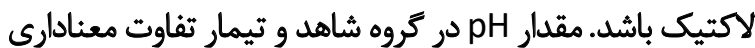

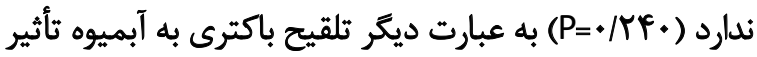

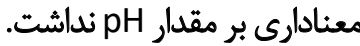

طبق جدول شماره | اسيديته نمونه تيمار در روز اول

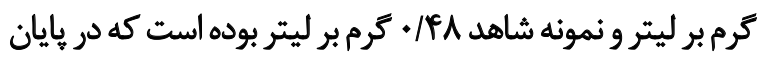

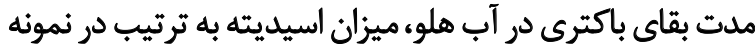

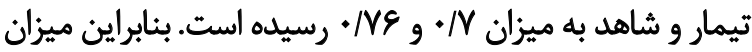

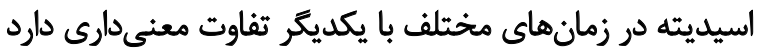

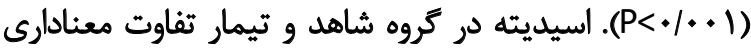

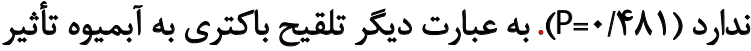

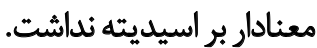

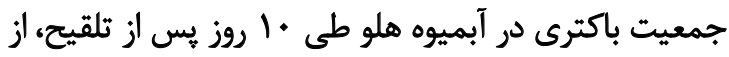

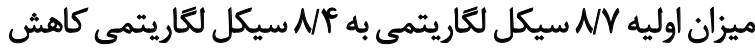

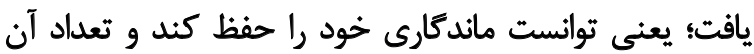

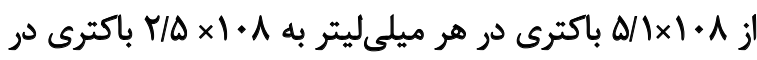

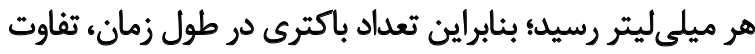

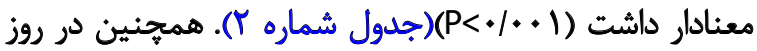

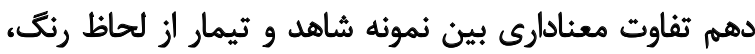

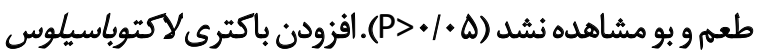

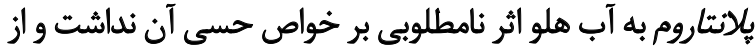

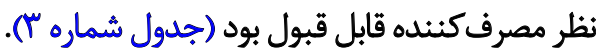

\section{بحث و نتيجنه كيرى}

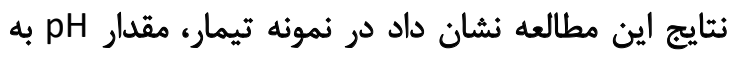

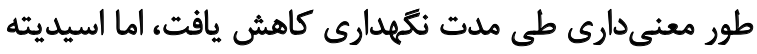

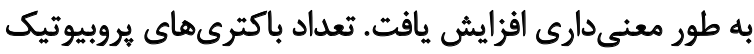

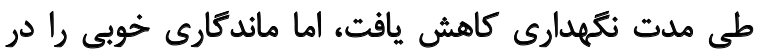

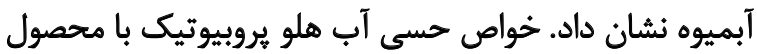

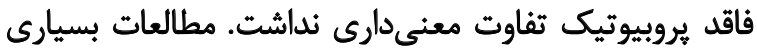

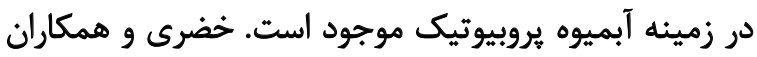

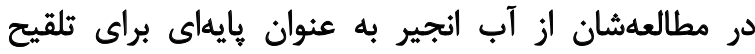

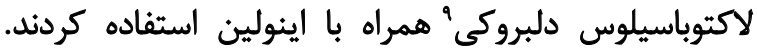

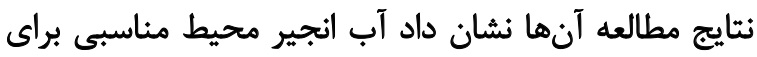

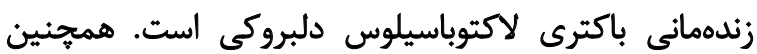

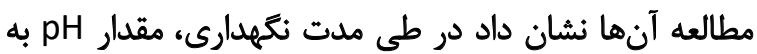

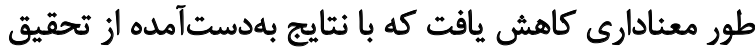

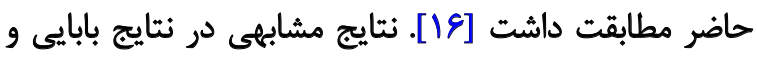

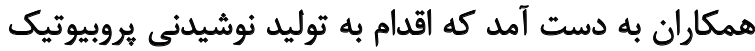

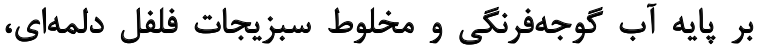

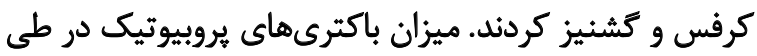

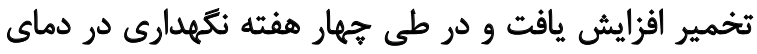

درنتيجه بعد از كذشت اين زمان، تودهاي كه نشاندهنده رشد ريد

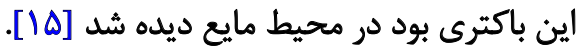

آبميوه هلو از شركت سنايج در ايران تهيه و وييش از استفاده

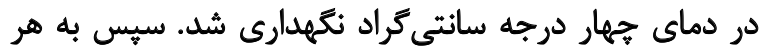

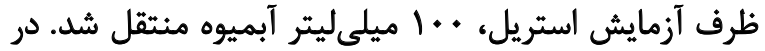

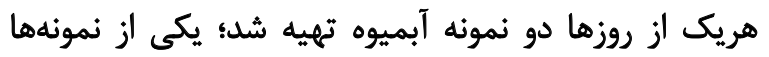

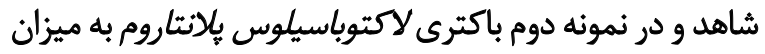

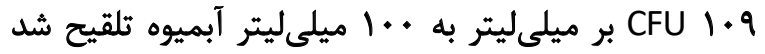

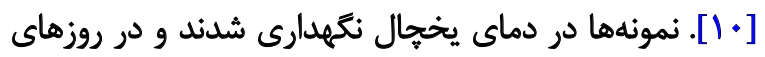

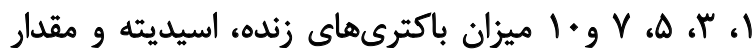

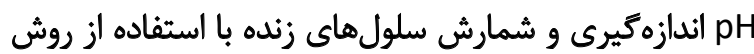

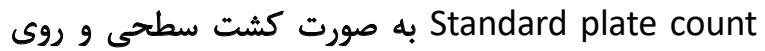

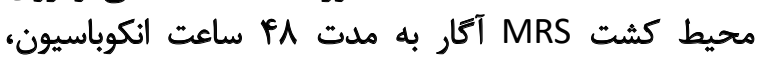

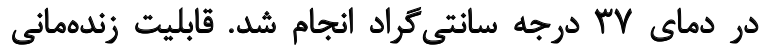

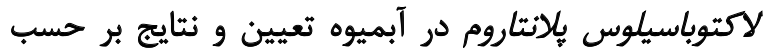

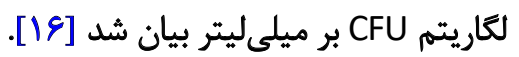

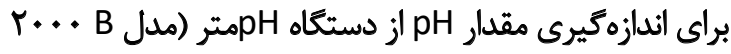

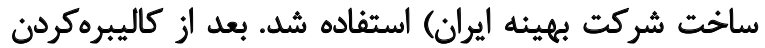

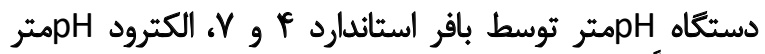

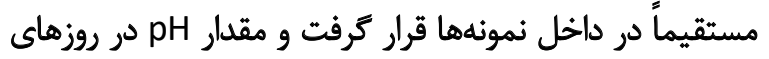

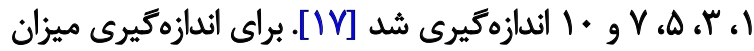

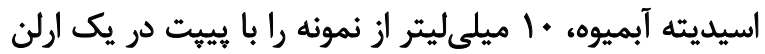

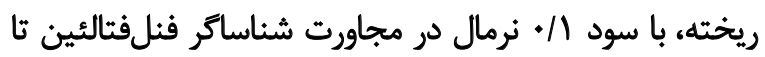

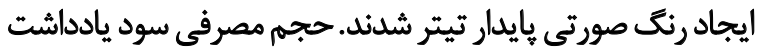

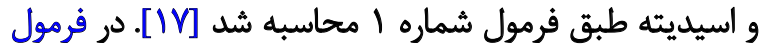

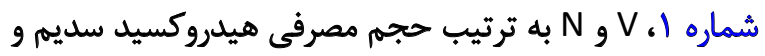
نرماليته سود مصرفى هستئل.

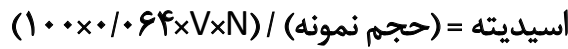

نمونههاى تيمار و شاهد در روز دهم با حضور هفت ارزياب

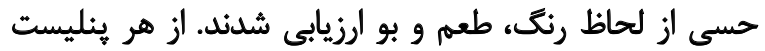

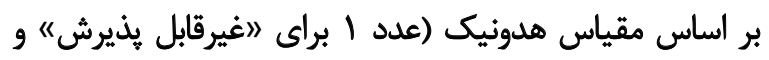

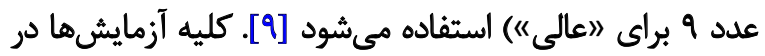

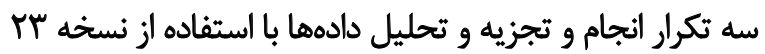
نرمافزار SPSS و آزمونهاي تى تى مستقل و و تحليل وانيل واريانس در

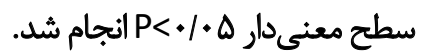

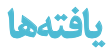

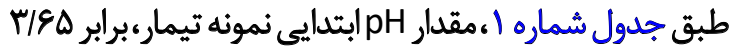

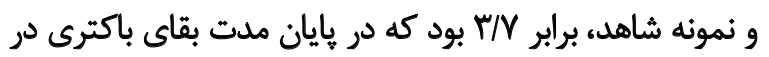

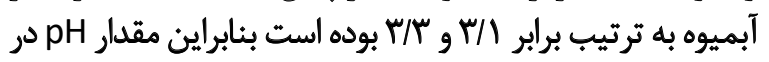

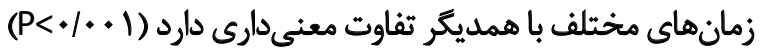




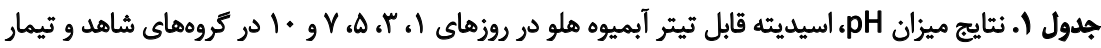

\begin{tabular}{|c|c|c|c|}
\hline \multicolumn{2}{|c|}{ كيووها } & \multirow{2}{*}{ زمان (روز) } & \multirow{2}{*}{ متغير } \\
\hline تيمار & شاهد & & \\
\hline$r / \& \Delta \pm . / \cdot \Delta^{\mathrm{aA}}$ & $r N \cdot \pm \cdot / /^{\mathrm{aA}}$ & 1 & \\
\hline$r / \Delta) \pm * / *)^{a B}$ & $r / \Delta Y \pm+A$ aB & $r$ & \\
\hline$r / R \vee \pm . /$. ६aB & $\Gamma / A T \pm \cdot / N^{\mathrm{aB}}$ & $\Delta$ & $\mathrm{pH}$ \\
\hline$r / T \cdot \pm \cdot / \cdot q b c$ & $r / f i \pm \cdot / \cdot \Delta^{a C}$ & $r$ & \\
\hline$m / 1 \cdot \pm \cdot 1 \cdot \Delta^{b c}$ & $r / r \cdot \pm \cdot / \cdot r a c$ & 1. & \\
\hline$\Delta \mathrm{V} / \cdot \pm+V_{0} \cdot \mathrm{aA}$ & - $/$ EA土./. YaA & 1 & \\
\hline$\cdot / 17 \pm \cdot 1 \cdot 1^{\mathrm{bB}}$ & 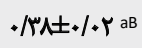 & $r$ & \\
\hline$+|\Delta| \pm+/+\Gamma_{\mathrm{bA}}$ & $\cdot / \Gamma+ \pm \cdot / \cdot r^{\mathrm{ma}}$ & $\Delta$ & اسيديته (درصد) \\
\hline$\cdot / \Delta V \pm / / r b c$ & $+(\Delta) \pm+/+)^{a c}$ & $\gamma$ & \\
\hline$\cdot N \cdot \pm * / * f a D$ & 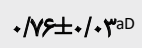 & 1. & \\
\hline
\end{tabular}

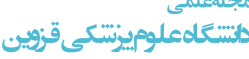

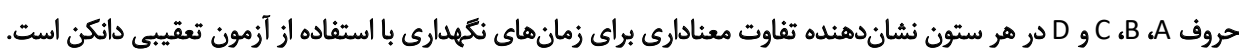

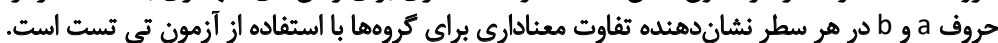

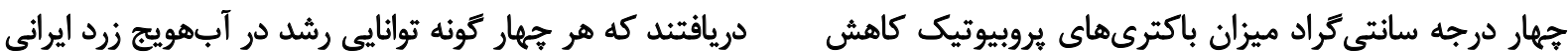

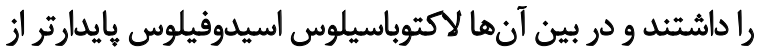

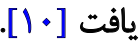

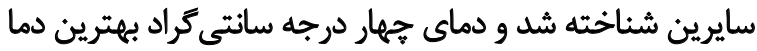

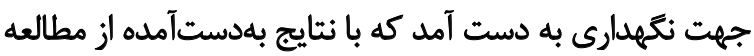
حاضر مطابقت دارد [19].

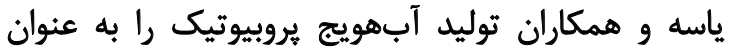

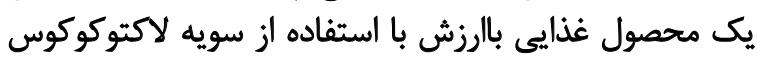

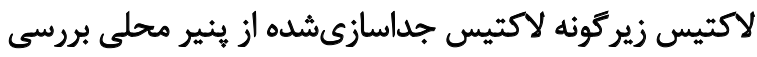

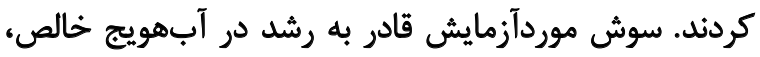

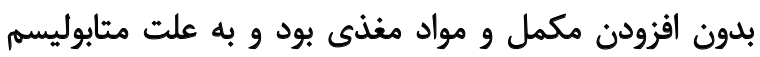

نتايج مطالعه اميدى و همكاران در بررسى اثر ضدديابتى

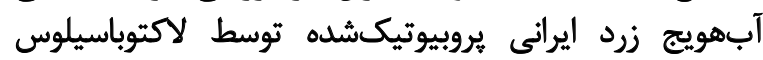

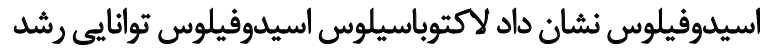

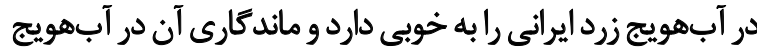

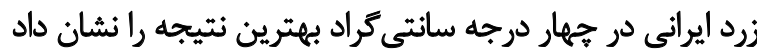

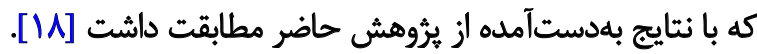

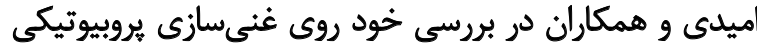

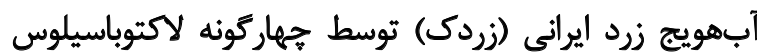

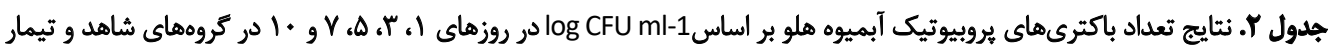

\begin{tabular}{|c|c|c|c|}
\hline \multicolumn{2}{|c|}{ كروهها } & \multirow{2}{*}{ زمان (روز) } & \multirow{2}{*}{ متغير } \\
\hline تيمار & شاهد & & \\
\hline$N V \cdot \pm+/ Y^{A}$ & - & 1 & \\
\hline$N 8 \cdot \pm \cdot N \cdot A B$ & - & $r$ & \\
\hline$N \widetilde{N} \pm \pm / N \Delta^{C}$ & - & $\Delta$ & شمارش باكترى \\
\hline$N \Delta+ \pm * / 1 \cdot A B C$ & - & $\gamma$ & \\
\hline$N+ \pm \pm * / M A^{B C}$ & - & 1. & \\
\hline
\end{tabular}

حروف مختلف A، B و C در هر ستون نشاندهنده تفاوت معنادارى براى زمانهاي نكَهدارى با استفاده از آزمون تعقيبي دانكن هستئد. 
جدول بّ. نتايج ارزيابى حسى آبميوه هلو در نمونههاي شاهد و تيمار

\begin{tabular}{|c|c|c|c|c|}
\hline \multirow{2}{*}{ مقدار احتمال } & \multicolumn{2}{|c|}{ كروها } & \multirow{2}{*}{\multicolumn{2}{|c|}{ مثغير }} \\
\hline & تيمار & شاهد & & \\
\hline • & $\mathrm{V} \pm \cdot(\lambda)^{\mathrm{a}}$ & $\mathrm{V} / \Delta \mathrm{V} \pm$ - $/ 9 \mathrm{Y}^{\mathrm{a}}$ & طعم & \\
\hline.$/ 19 \Delta$ & $\vee / T Q \pm \cdot / 9 \Delta^{a}$ & 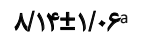 & رن & \\
\hline $1 / \ldots$ & $V / r 9 \pm \cdot / 9 \Delta)^{a}$ & V/TQ土 & بو & ارزيابي حسى \\
\hline +/TAq & $V / 19 \pm+/ M^{\text {ea }}$ & $V / \& \& \pm \cdot / N^{a}$ & يذيرش كلى & \\
\hline
\end{tabular}

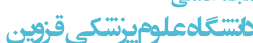

نشان داده است [Q] كه نتايج اين مطالعه با مطالعه حاضر

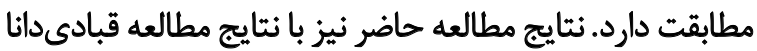

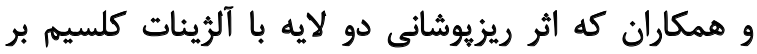
زندهمانى باكترى لاكتوباسيلوس اسيدوفيلوس، طي دورئ دوره نكهدارى آب كوجهفرنكى را بررسى كرده بود و كروه ارئيابان

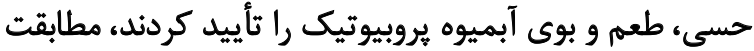

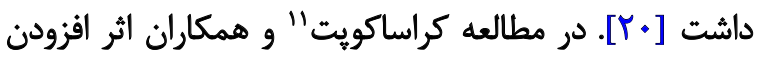

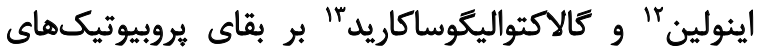

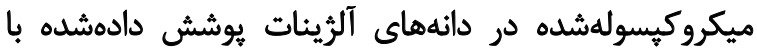

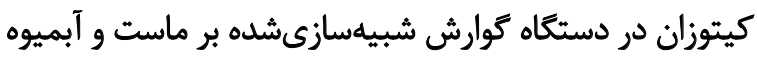

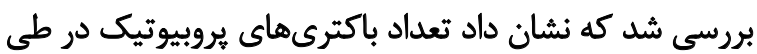

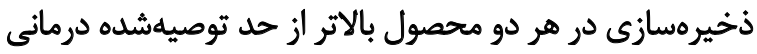

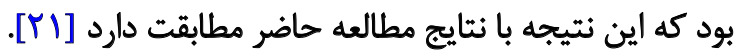

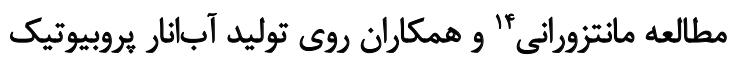

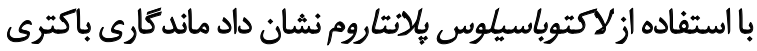

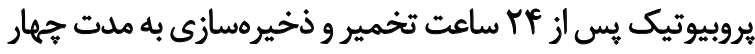

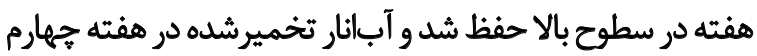
ذخيرهسازى بهتر از آبانار غيرتخميرشده بود كه اين مطالعه نيز نتايج مشابههى با مطالعه حاضر داشت [YrT].

در اين يرؤهش با توجه به نتايج آزمون هاي انجامشيده مشاهده

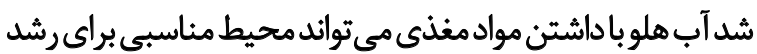

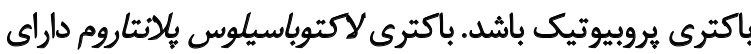

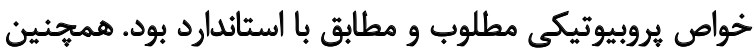

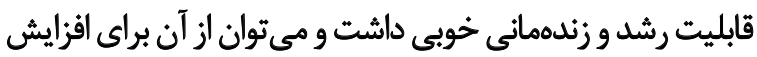

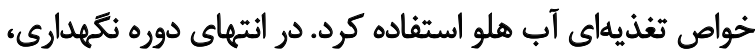

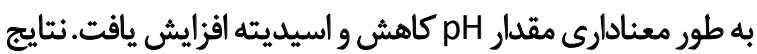

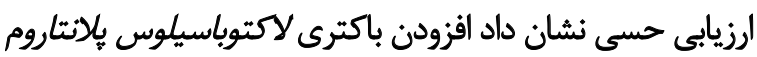

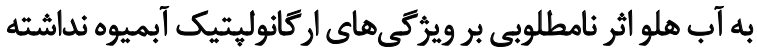
است؛ به كونهاي كه در يايان مطالعه رئك، طعهم و بوى آبميوه

\section{Krasaekoopt}

12. Inulin

13. Galactooligosaccharide

14. Mantzourani
شديد توانست مقدار pH آبهويج رانتا زير جهار كاهش دهد

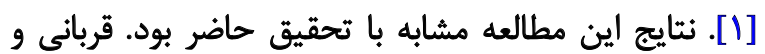

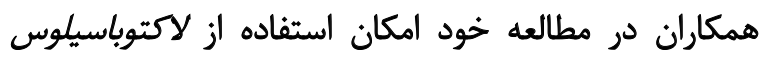

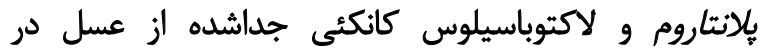

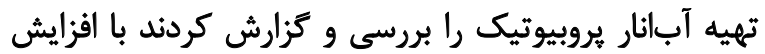

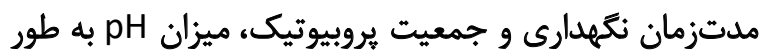

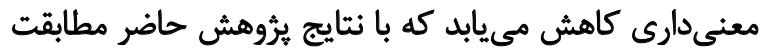

دارد [11] معني

نتايج مطالعه توتونجي و همكاران نشان داد لاكتوباسيلوس

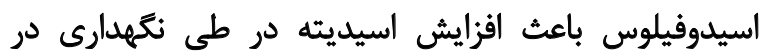

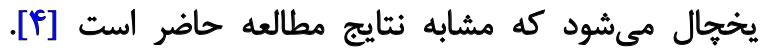

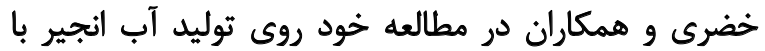

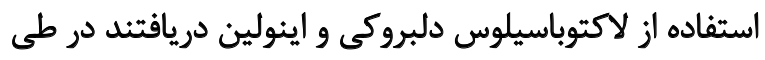

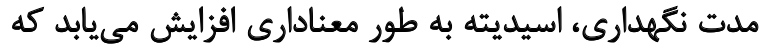

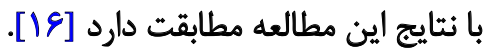
برخلاف نتايج حاصل از تحقيق حاضر، نتايج تحقيقاث قضاوى

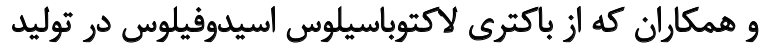

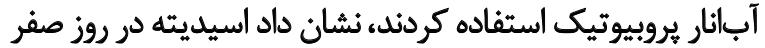

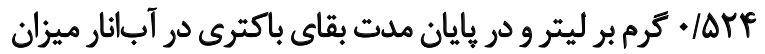

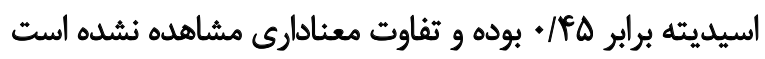

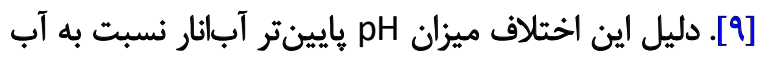

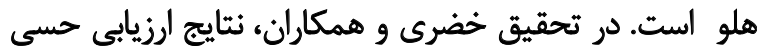

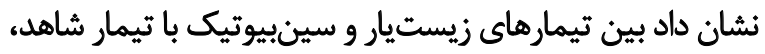

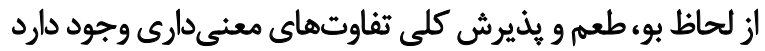

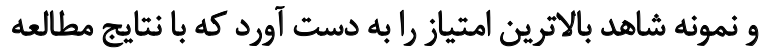

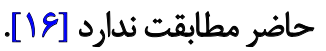

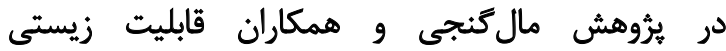

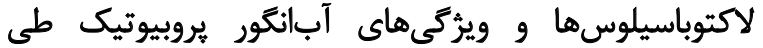

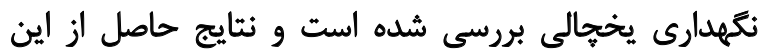

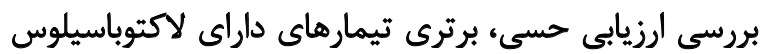

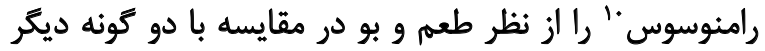

10. Lactobacillus rhamnosus 
حالت طبيعى داشت واز نظر مصرفكنينده قابل قبول بود. با توليد

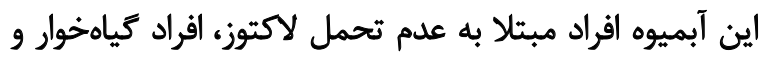

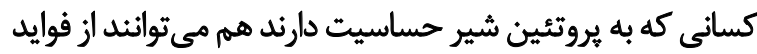
محصولات يروبيوتيك بهرهمند شوند.

مالاحظات الخالاقي بيروى أز اصول اخلاق ثبروهش مقاله حاضر مستخرج از طرح دانشجويى مصوب دانشعاه

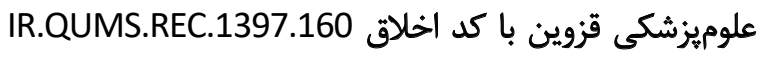

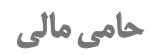

تحقيق حاضر با حمايت مالى معاونت محترم يثروهشى دانشعاه

$$
\text { علوميزشكى قزوين صورت كرفته است. }
$$

$$
\text { مشار كت نويسندكًان }
$$

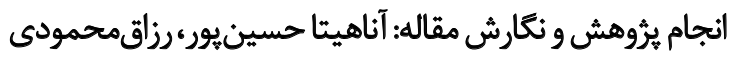
و سعيد شهسوارى؛ تحليل دادها: آناهيتا حسينيونيور، سعيد

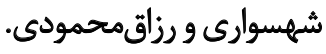

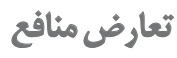

بنابر اظهار نويسندكان اين مقاله تعارض منافع ندارد.

$$
\text { تشكر و قدرداثي }
$$

بدينوسيله از معاونت يُروهشى علوم يزشكى دانشكاه قزوين تقدير و تشكر مى بـوسيل ازمعانت 


\section{References}

[1] Ayaseh A, Taban H, Yari Khosroshahi A. Production of probiotic carrot juice with using of Lactococcus lactis. Food Ind Res. 2017; 27 (4):183-91. [In Persian]

[2] Malganji S, Eivani M, Sohrabvandi S, Mortazavian A. Health related aspects of probiotics. Iran J Nut Sci Food Tech. 2013; 7(5):580-90. [In Persian]

[3] Kakebaveh M, Taheri HR, Harki Nejad T. Effects of dietary supplementation with probiotics, prebiotic and synbiotic on performance and intestinal microbial population of broiler chickens. J Res Anim Protect. 2014; 5(10):44-56. [In Persian]

[4] Totonchi P, Hesari J, Moradi M, Fathi AB. production and evaluation of probiotic red grape juice by latobacillus acidophilus La5, and lactobacillus casel. J Food Res. 2015; 25(4):656-66. [In Persian]

[5] Malganji SH, Jahadi M, Shadnoush M, Mortazavian AM, Sohrabvandi S, Eivani MJ. Studying of viability of Lactobacillus and organoleptic properties probiotic grape juice during cold storage. J Med Refinement. 2014; 23(2):124-32. [In Persian]

[6] Ghasemkhani H, Sobhanardakani S, Cheraghi M. Health risk assessment of consumption of commercial fruit juices marketed in Hamedan City based on potential risk of $\mathrm{Zn}$ and $\mathrm{Cd}$. $J$ Neyshabur Univ Med Sci. 2016; 4(2):32-40. [In Persian]

[7] Usefi A, Shahi F, Shekhlui H. Investigating the behavior of the shear stream of Iranian peach juice: Effect of concentration and temperature. Q J Mod Food Tech. 2015; 3(10):11-22. [In Persian]

[8] Abdi F, Atarodi KZ, Mirmiran P, Esteki T. Surveying global and Iranian food consumption patterns: A review of the literature. J Fasa Univ Med Sci. 2015; 5(2):159-67. [In Persian]

[9] Ghazavi N, Abedi R. Using lactobacillus acidophilus in production of probiotic pomegranate juice. Food Sci Tech. 2018; 15(77):107-99. [In Persian]

[10] Babaei M, Hashemiravan M, Pourahmad R. Production of probiotic beverage based on tomato juice and mixture of sweet pepper, celery and coriander juices. Iran J Nut Sci Food Tech. 2018; 15 (74):341-31. [In Persian]

[11] Ghorbani N, Nateghi L, Tajabadi N. Investigating the possibility of using Lactobacillus plantarum and Lactobacillus kunkeei isolated from honey in the preparation of probiotic pomegranate juice. Iran J Nut Sci Food Tech. 2018; 15(76):23-13. [In Persian]

[12] Khamirlan R, Jooyandeh J, Hesari J, Barzegar H. optimization and investigation on physicochemical, microblal and sensory quality of permeate-based probiotic orange beverage. Iran J Food Sci Tech. 2017; 14(65):185-97. [In Persian]

[13] Nematollahi A, Sohrabvandi S, Farsani A, Komeyi R, Asadzade S. Studying the effect of refrigerated storage on the viability of native and industrial probiotic strains and some physicochemical and sensory properties in cornelian cherry juice. Iran J Food Sci Tech. 2015; 9(4):87-96. [In Persian]

[14] Chalabi PSM, Shahin Z. Investigating the physicochemical properties of apple juice and probiotic banana during main- tenance. Paper presented: $3^{\text {rd }}$ National Conference on Food Science and Technology. 14 September 2014; Quchan, Iran. [In Persian]

[15] Dogahe M, Towfighi A, Khosravi-Darani K, Dadgar M, Mortazavian A, Ahmadi N. Influence of pomegranate peel on viability of probiotic bacteria in pomegranate juice Iran J Nut Sci Food Tech. 2013; 7(5):17-24. [In Persian]

[16] Khezri S, Mahmoudi R, Dehghan P. Fig juice fortified with inulin and Lactobacillus Delbrueckii: A promising functional food. J Appl Food Biomed. 2018; 5(2):97-106. [In Persian]

[17] Institute of Standards and Industrial Research of Iran. Fruit juice-(2007). Test methods, Iranian National Standard No. 2685. Tehran: Institute of Standards and Industrial Research of Iran; 2007.

[18] Omidi B, Fazeli MR, Amozegar MA, Mortazavi P. Antidiabetic effect of probioticated persian yellow carrot juice with Lactobacillus acidophilus. Comp Pathobiol. 2011; 8(1):395-402. [In Persian]

[19] Omidi B, Fazeli M, Amuzegar M, Jamali Far H. Probiotic enrichment of Iranian Yellow Carrot (Zardak) by four species of Lactobacillus. J Knowledge Microbiol. 2010; 2(6):51-9. [In Persian]

[20] Ghobadi M , Rashinadi T. Evaluation of the effect of two-layer microsphere effect with calcium alginate on the lactobacillus acidophilus survival during tomato wine storage. New food Tech. 2016; 4(2):151-60. [In Persian]

[21] Krasaekoopt W, Watcharapoka S. Effect of addition of inulin and galactooligosaccharide on the survival of microencapsulated probiotics in alginate beads coated with chitosan in simulated digestive system, yogurt and fruit juice. LWT-Food Sci Tech. 2014; 57(2):761-6. [DOI:10.1016/j.Iwt.2014.01.037]

[22] Mantzourani I, Kazakos S, Terpou A, Alexopoulos A, Bezirtzoglou $E$, Bekatorou $A$, et al. Potential of the probiotic lactobacillus plantarum ATCC 14917 strain to produce functional fermented pomegranate juice. Foods. 2019; 8(1):1-13. [DOI:10.3390/foods8010004] [PMID] [PMCID] 